\title{
Front Matter: Volume 8552
}

, "Front Matter: Volume 8552," Proc. SPIE 8552, Semiconductor Lasers and Applications V, 855201 (14 December 2012); doi: 10.1117/12.1518468

SPIE. Event: Photonics Asia, 2012, Beijing, China 


\section{Photomask Technology 2012}

Frank E. Abboud

Thomas B. Faure

Editors

11-13 September 2012

Monterey, California, United States

Sponsored by

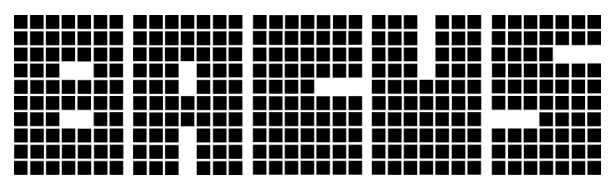

The international technical group of SPIE dedicated

to the advancement of photomask technology

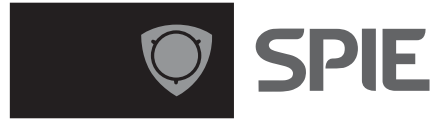

Published by

SPIE

Volume 8522 
The papers included in this volume were part of the technical conference cited on the cover and title page. Papers were selected and subject to review by the editors and conference program committee. Some conference presentations may not be available for publication. The papers published in these proceedings reflect the work and thoughts of the authors and are published herein as submitted. The publisher is not responsible for the validity of the information or for any outcomes resulting from reliance thereon.

Please use the following format to cite material from this book:

Author(s), "Title of Paper," in Photomask Technology 2012, edited by Frank E. Abboud, Thomas B. Faure, Proceedings of SPIE Vol. 8522 (SPIE, Bellingham, WA, 2012) Article CID Number.

ISSN: 0277-786X

ISBN: 9780819492609

Published by

SPIE

P.O. Box 10, Bellingham, Washington 98227-0010 USA

Telephone +1 3606763290 (Pacific Time) · Fax +1 3606471445

SPIE.org

Copyright $@ 2012$, Society of Photo-Optical Instrumentation Engineers.

Copying of material in this book for internal or personal use, or for the internal or personal use of specific clients, beyond the fair use provisions granted by the U.S. Copyright Law is authorized by SPIE subject to payment of copying fees. The Transactional Reporting Service base fee for this volume is $\$ 18.00$ per article (or portion thereof), which should be paid directly to the Copyright Clearance Center (CCC), 222 Rosewood Drive, Danvers, MA 01923. Payment may also be made electronically through CCC Online at copyright.com. Other copying for republication, resale, advertising or promotion, or any form of systematic or multiple reproduction of any material in this book is prohibited except with permission in writing from the publisher. The CCC fee code is 0277-786X/12/\$18.00.

Printed in the United States of America.

Publication of record for individual papers is online in the SPIE Digital Library.

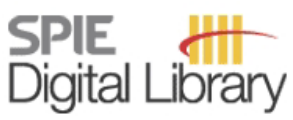

SPIEDigitalLibrary.org

Paper Numbering: Proceedings of SPIE follow an e-First publication model, with papers published first online and then in print and on CD-ROM. Papers are published as they are submitted and meet publication criteria. A unique, consistent, permanent citation identifier (CID) number is assigned to each article at the time of the first publication. Utilization of CIDs allows articles to be fully citable as soon as they are published online, and connects the same identifier to all online, print, and electronic versions of the publication. SPIE uses a six-digit CID article numbering system in which:

- The first four digits correspond to the SPIE volume number.

- The last two digits indicate publication order within the volume using a Base 36 numbering

system employing both numerals and letters. These two-number sets start with 00, 01, 02, 03, 04, 05, 06, 07, 08, 09, 0A, OB ... 0Z, followed by 10-1Z, 20-2Z, etc.

The CID Number appears on each page of the manuscript. The complete citation is used on the first page, and an abbreviated version on subsequent pages. Numbers in the index correspond to the last two digits of the six-digit CID Number. 


\title{
Contents
}

\author{
xiii Conference Committee \\ xvii Introduction \\ xix Schedule of Special Session: Will optical patterning solutions be ready if EUV lithography \\ continues to be delayed?
}

\section{SESSION 1 KEYNOTE SESSION}

852202 Transform designs to chips, an end user point of view on mask making (Keynote Paper) [8522-1]

J. Y. Chen, NVIDIA Corp. (United States)

\section{SESSION 2 INVITED SESSION}

$852203 \quad 2012$ Mask Industry Survey [8522-2]

M. Malloy, L. C. Litt, SEMATECH (United States)

\section{SESSION 3 PATTERNING}

852205 Improving CD uniformity using MB-MDP for 14nm node and beyond [8522-4]

B. G. Kim, J. Choi, J. Park, C. U. Jeon, SAMSUNG Electronics Co., Ltd. (Korea, Republic of);

S. Watson, A. Adamov, B. Pack, I. Bork, D2S, Inc. (United States)

852206 Impact of an etched EUV mask black border on imaging and overlay [8522-5]

N. Davydova, R. de Kruif, ASML Netherlands B.V. (Netherlands); N. Fukugami, S. Kondo, Toppan Printing Co., Ltd. (Japan); V. Philipsen, IMEC (Belgium); E. van Setten, ASML Netherlands B.V. (Netherlands); B. Connolly, Toppan Photomasks, Inc. (Germany); A. Lammers, V. Vaenkatesan, ASML Netherlands B.V. (Netherlands); J. Zimmerman, N. Harned, ASML Wilton (United States)

852207 An enhanced measure of mask quality using separated models [8522-6] A. Adamov, B. Pack, D2S, Inc. (United States); K. Hagiwara, D2S Japan (Japan), I. Bork, D2S, Inc. (United States); J. Choi, J. Park, B.-G. Kim, SAMSUNG Electronics Co., Ltd. (Korea, Republic of)

852208 Cold-development tool and technique for the ultimate resolution of ZEP520A to fabricate an EB master mold for nano-imprint lithography for 1Tbit/inch2 BPM development [8522-7] H. Kobayashi, H. Iyama, T. Kagatsume, T. Watanabe, HOYA Corp. (Japan) 
852209 Improvement of lithographic performance and reduction of mask cost by simple OPC [8522-8]

K. Tsujita, K. Mikami, H. Ishii, T. Arai, R. Nakayama, Canon Inc. (Japan); M. C. Smayling, Tela Innovations, Inc. (United States); V. Axelrad, Sequoia Design Systems, Inc. (United States); H. Yaegashi, K. Oyama, Tokyo Electron, Ltd. (Japan)

8522 OA A profile-aware resist model with variable threshold [8522-9]

S. Moulis, V. Farys, STMicroelectronics (France); J. Belledent, R. Thérèse, CEA-LETI (France);

S. Lan, Q. Zhao, M. Feng, L. Depre, R. Dover, ASML Brion Technologies, Inc. (United States)

8522 OB CD control with defect inspection: you can teach an old dog a new trick [8522-10] C. Utzny, A. Ullrich, J. Heumann, E. Mohn, S. Meusemann, Advanced Mask Technology Ctr. GmbH Co. KG (Germany); R. Seltmann, GLOBALFOUNDRIES Dresden (Germany)

8522 0C Study of critical dimension uniformity (CDU) using a mask inspector [8522-11] M.-C. Lin, C.-F. Yu, M.-T. Lai, L. T. H. Hsu, A. Chin, A. Yen, Taiwan Semiconductor Manufacturing Co. Ltd. (Taiwan)

8522 OD Correcting image placement errors using registration control (RegC) technology in the photomask periphery [8522-84]

A. Cohen, Carl Zeiss SMS Ltd. (Israel); F. Lange, Advanced Mask Technology Ctr. (Germany); G. Ben-Zvi, E. Graitzer, D. Vladimir, Carl Zeiss SMS Ltd. (Israel)

8522 OE Impact of mask CDU and local CD variation on intra-field CDU [8522-13] J. Miyazaki, ASML Japan Co., Ltd. (Japan); O. Mouraille, J. Finders, ASML Netherlands B.V. (Netherlands); M. Higuchi, Y. Kojima, S. Sato, H. Morimoto, Toppan Printing Co., Ltd. (Japan)

8522 OF Correlation between reticle- and wafer-CD difference of multiple $28 \mathrm{~nm}$ reticle-sites [852214] G. Ning, GLOBALFOUNDRIES Dresden (Germany) and GLOBALFOUNDRIES Malta (United States); F. Richter, T. Thamm, GLOBALFOUNDRIES Dresden (Germany); P. Ackmann, GLOBALFOUNDRIES Malta (United States); M. Staples, F. Weisbuch, K. Kurth, J. Schenker, A. Leschok, GLOBALFOUNDRIES Dresden (Germany); F. N. GN, GLOBALFOUNDRIES Singapore Pte. Ltd. (Singapore)

8522 OG Comparison of critical dimension measurements of a mask inspection system with a CDSEM [8522-85]

J. P. Heumann, A. Ullrich, C. S. Utzny, S. Meusemann, F. Kromer, Advanced Mask Technology Ctr. GmbH Co. KG (Germany); J. M. Whittey, E. Garcia, M. Wagner, KLA-

Tencor Corp. (United States); N. J. Schmidt, KLA-Tencor Germany (Germany)

\section{SESSION $5 \quad$ MASK INSPECTION AND REPAIR I}

$8522 \mathrm{OH}$ Impact of EUVL mask surface roughness on an actinic blank inspection image and a wafer image [8522-16]

T. Yamane, T. Terasawa, EUVL Infrastructure Development Ctr., Inc. (Japan)

iv 
8522 ol Illuminating EUVL mask defect printability [8522-17]

K. D. Badger, Z. J. Qi, E. Gallagher, IBM Microelectronics (United States); K. Seki, Toppan Photomasks, Inc. (United States); G. McIntyre, IBM Microelectronics (United States)

8522 0J EUV multilayer defect compensation (MDC) by absorber pattern modification: improved performance with deposited material and other progresses [8522-18]

L. Pang, M. Satake, Y. Li, P. Hu, V. Tolani, D. Peng, D. Chen, B. Gleason, Luminescent Technologies, Inc. (United States)

8522 OK Capability of model EBEYE M for EUV mask production [8522-19]

M. Naka, S. Yamaguchi, M. Kadowaki, T. Koike, T. Hirano, M. Itoh, Y. Yamazaki, Toshiba Corp. (Japan); K. Terao, M. Hatakeyama, K. Watanabe, H. Sobukawa, T. Murakami, K. Tsukamoto, T. Hayashi, R. Tajima, N. Kimura, EBARA Corp. (Japan); N. Hayashi, Dai Nippon Printing Co., Ltd. (Japan)

$8522 \mathrm{OL}$ Electron beam inspection of 16nm HP node EUV masks [8522-20]

T. Shimomura, S. Narukawa, T. Abe, T. Takikawa, N. Hayashi, Dai Nippon Printing Co., Ltd. (Japan); F. Wang, L. Ma, C.-W. Lin, Y. Zhao, C. Kuan, J. Jau, Hermes-Microvision Inc. (United States)

8522 OM EUV mask inspection study for sub-20nm device [8522-21]

I. Shin, G. Yoon, J. H. Na, P. D. H. Chung, C. Jeon, SAMSUNG Electronics Co., Ltd. (Korea, Republic of)

\section{SESSION 6 MATERIAL AND PROCESS}

$8522 \mathrm{ON}$ The E-beam resist test facility: performance testing and benchmarking of E-beam resists for advanced mask writers (Invited Paper) [8522-22]

M. Malloy, I. Y. Jang, M. Mellish, L. C. Litt, SEMATECH (United States); A. Raghunathan,

J. Hartley, College of Nanoscale Science \& Engineering (United States)

852200 Conductive layer for charge dissipation during electron-beam exposures [8522-23]

L. D. Bozano, R. Sooriyakumaran, IBM Almaden Research Ctr. (United States); T. Nagasawa,

S. Watanabe, Y. Kawai, Shin-Etsu Chemical Co., Ltd. (Japan); S. Kondo, J. Kotani,

M. Kagawa, Toppan Printing Co., Ltd. (Japan); L. K. Sundberg, M. I. Sanchez, E. M. Lofano,

C. T. Rettner, IBM Almaden Research Ctr. (United States); T. Senna, Toppan Photomasks,

Inc. (United States); T. Faure, IBM Corp. (United States)

8522 OP Mask characterization for CDU budget breakdown in advanced EUV lithography [8522-24]

P. Nikolsky, C. Strolenberg, R. Nielsen, T. Nooitgedacht, N. Davydova, G. Yang, S. Lee, ASML Netherlands B.V. (Netherlands); C.-M. Park, I. Kim, J.-H. Yeo, SAMSUNG Electronics Co., Ltd.

(Korea, Republic of)

$85220 Q \quad$ Process challenges in advanced photomask etch processes [8522-25]

C. J. Choi, K. Yung, C.-H. Ma, G. Vanamu, Intel Corp. (United States)

8522 OR Advanced photomask fabrication process to increase pattern reliability for sub-20nm node [8522-26]

D. I. Shin, S. J. Jo, H. Y. Jang, Y. K. Hong, D. H. Hwang, C. S. Choi, D. S. Jang, H. Y. Jung,

T. J. Ha, S. P. Kim, D. G. Yim, SK Hynix Inc. (Korea, Republic of) 
8522 OS Study and comparison of negative tone resists for fabrication of bright field masks for $14 \mathrm{~nm}$ node [8522-27]

A. E. Zweber, T. Faure, A. McGuire, IBM Corp. (United States); L. K. Sundberg,

R. Sooriyakumaran, M. I. Sanchez, L. D. Bozano, IBM Almaden Research Ctr. (United States);

T. Senna, Y. Fujita, Y. Negishi, Toppan Photomasks, Inc. (United States); M. Tanabe,

T. Kaneko, Toppan Printing Co., Ltd. (Japan)

\section{SESSION 7 MASK DATA PREPARATION I}

8522 OT Novel DPT methodology co-optimized with design rules for sub-20nm device [8522-28]

H.-J. Lee, S.-H. Choi, J.-S. Yang, K.-Y. Chun, J. Do, C.-H. Park, SAMSUNG Electronics Co., Ltd. (Korea, Republic of)

8522 OU Mask design automation: an integrated approach [8522-29]

R. Gladhill, P. Buck, A. Wong, Toppan Photomasks, Inc. (United States)

8522 OV Generating well-behaved OASIS files for mask data processing [8522-30]

D. Hung, J. P. Canepa, K. Kuo, Synopsys, Inc. (United States); J. G. Jou, Taiwan

Semiconductor Manufacturing Co. Ltd. (Taiwan)

8522 OW Enhancement of correction for mask process through dose correction on already geometrically corrected layout data [8522-77]

M. M. Reddy, B. D. S. S., N. Rao, A. Rajagopalan, R. R. Pai, SoftJin Technologies Pvt. Ltd. (India)

8522 OX Automatic marking by use of MRCC range pattern matching for advanced MDP [8522-32] D. Salazar, W. Moore, J. Valadez, Synopsys, Inc. (United States)

\section{SESSION $8 \quad$ SIMULATION AND MODELING}

8522 OY Choosing the data flow paradigm for EUV mask process corrections [8522-33]

C. Bürgel, Advanced Mask Technology Ctr. (Germany); K. Standiford, GLOBALFOUNDRIES (United States); G. S. Chua, GLOBALFOUNDRIES Singapore (Singapore)

$85220 Z$ Bridging the gaps between mask inspection/review systems and actual wafer printability using computational metrology and inspection (CMI) technologies [8522-34] L. Pang, V. Tolani, M. Satake, P. Hu, D. Peng, T. Liu, D. Chen, B. Gleason, A. Vacca, Luminescent Technologies (United States)

852210 Particle transport in plasma systems for development of EUVL mask blanks [8522-104] P. Stoltz, A. Likhanskii, C. Zhou, Tech-X Corp. (United States); V. Jindal, P. Kearney, SEMATECH North (United States)

852211 Interactions of 3D mask effects and NA in EUV lithography (Second Place, Best Paper Award) [8522-107]

J. T. Neumann, P. Gräupner, W. Kaiser, R. Garreis, Carl Zeiss SMT GmbH (Germany); B. Geh, Carl Zeiss SMT Inc. (United States) 
852212 Advanced module for model parameter extraction using global optimization and sensitivity analysis for electron beam proximity effect correction [8522-37]

T. Figueiro, ASELTA Nanographics (France) and CNRS/UJF-Grenoble 1/CEA LTM (France); K.-H. Choi, M. Gutsch, M. Freitag, C. Hohle, Fraunhofer Ctr. Nanoelectronic Technologies (Germany); J.-H. Tortai, CNRS/UJF-Grenoblel/CEA LTM (France); M. Saib, P. Schiavone, ASELTA Nanographics (France)

\section{SESSION 9 CLEANING/CONTAMINATION/HAZE}

852213 Sub-20-nm node photomask cleaning enhancement by controlling zeta potential (Invited Paper) [8522-38]

K.-W. Lin, C.-L. Lu, C.-W. Shen, L. Hsu, A. Chin, A. Yen, Taiwan Semiconductor Manufacturing Co. Ltd. (Taiwan)

852214 Effect of radiation exposure on the surface adhesion of Ru-capped MoSi multilayer blanks (First Place, Best Poster Award) [8522-39]

G. Durkaya, A. Rastegar, H. Kurtuldu, SEMATECH (United States)

852215 The plasma etching methods for minimizing mask CD variation by cleaning process [852240]

H. D. Shin, S. K. Jeong, H. Y. Jung, S. P. Kim, D. G. Yim, SKHynix Inc. (Korea, Republic of)

852216 Preparation of substrates for EUV blanks using an etch clean process to meet HVM challenges [8522-41]

A. J. Kadaksham, R. Teki, J. Harris-Jones, C. C. Lin, SEMATECH (United States)

852217 Controlling MegaSonic performance by optimizing cleaning media's physical and gaseous properties [8522-61]

H. Shende, MP Mask Technology Ctr., LLC (United States); S. Singh, SUSS MicroTec Inc. (United States); J. Baugh, MP Mask Technology Ctr., LLC (United States); U. Dietze, SUSS MicroTec Inc. (United States); P. Dress, SUSS MicroTec Photomask Equipment GmbH \& Co. KG (Germany)

852218 A new approach in dry technology for non-degrading optical and EUV mask cleaning [8522-43]

I. Varghese, B. Smith, M. Balooch, C. Bowers, Eco-Snow Systems, Rave N.P. Inc. (United States)

\section{SESSION 10 SOURCE/MASK OPTIMIZATION}

852219 The significance of rigorous electromagnetic field simulation on mask development for 20nm optical lithography technology [8522-44] F. Jiang, Y. Deng, J. Kye, H. J. Levinson, P. Ackmann, GLOBALFOUNDRIES Inc. (United States); B. I. Choi, GLOBALFOUNDRIES Inc. (Singapore); F. Schurack, M. Sczyrba, Advanced Mask Technology Ctr. GmbH Co. KG (Germany) 
$85221 \mathrm{~A}$ The new test pattern selection method for OPC model calibration, based on the process of clustering in a hybrid space [8522-45]

D. Vengertsev, K. Kim, S.-H. Yang, S. Shim, S. Moon, SAMSUNG Electronics Co., Ltd. (Korea, Republic of); A. Shamsuarov, Graphics Solution Group (Russian Federation); S. Lee, S.-W. Choi, J. Choi, H.-K. Kang, SAMSUNG Electronics Co., Ltd. (Korea, Republic of)

8522 1B OPC and verification for LELE double patterning [8522-46]

K. Arb, C. Reid, Q. Li, E. Levine, P. Ghosh, Mentor Graphics Corp. (United States)

\section{SESSION 11 MASK LONG-TERM DURABILITY}

8522 1D Photomask film degradation effects in the wafer fab: how to detect and monitor over time [8522-48]

J. Whittey, C. Hess, E. Garcia, M. Wagner, B. Duffy, KLA-Tencor Corp. (United States)

8522 IE Reticle storage in microenvironments with extreme clean dry air [8522-49]

A. Gettel, D. Glüer, GLOBALFOUNDRIES Dresden(Germany); A. Honold, InReCon AG

(Germany)

\section{SESSION 12 MASK PATTERN GENERATORS}

$85221 G \quad$ Proposal of an extended loading effect correction for EBM-8000 [8522-51]

H. Matsumoto, Y. Kato, N. Nakayamada, S. Yoshitake, K. Hattori, NuFlare Technology, Inc. (Japan)

$8522 \mathrm{lH} \quad$ Printing results of a proof-of-concept $50 \mathrm{keV}$ electron multi-beam mask exposure tool (eMET POC) [8522-52]

E. Platzgummer, C. Klein, H. Loeschner, IMS Nanofabrication AG (Austria)

852211 Shape-dependent dose margin correction using model-based mask data preparation [8522-53]

Y. Kimura, R. Yamamoto, T. Kubota, K. Kouno, HOYA Corp. (Japan); K. Hagiwara,

S. Matsushita, D. Hara, D2S K.K. (Japan)

$85221 \mathrm{~J} \mathrm{Reflective} \mathrm{electron-beam} \mathrm{lithography} \mathrm{performance} \mathrm{for} \mathrm{the} \mathrm{10nm} \mathrm{logic} \mathrm{node} \mathrm{[8522-54]}$

R. Freed, T. Gubiotti, J. Sun, A. Cheung, J. Yang, M. McCord, P. Petric, A. Carroll,

U. Ummethala, L. Hale, J. Hench, S. Kojima, W. Mieher, C. F. Bevis, KLA-Tencor Corp. (United States)

$85221 \mathrm{~K}$ Future mask writers requirements for the sub-10nm node era (First Place, Best Paper Award) [8522-55]

M. Chandramouli, F. Abboud, N. Wilcox, A. Sowers, D. Cole, Intel Corp. (United States) 
8522 IL EUVL mask repair: expanding options with nanomachining (Third Place, Best Paper Award) [8522-56]

E. Gallagher, G. McIntyre, M. Lawliss, IBM Corp. (United States); T. Robinson, R. Bozak,

R. White, J. LeClaire, RAVE LLC (United States)

$85221 \mathrm{M}$ Ebeam based mask repair as door opener for defect free EUV masks [8522-57]

M. Waiblinger, T. Bret, Carl Zeiss SMS GmbH (Germany); R. Jonckheere, D. Van den Heuvel, IMEC (Belgium)

852210 Computational defect review for wafer-fab reticle requal, part 1: mask plane inspections [8522-59]

V. Tolani, G. Dai, S. Lakkapragada, K. Wang, P. Hu, L. He, Y. Li, D. Peng, G. Hwa,

L. Pang, Luminescent Technologies, Inc. (United States)

POSTER SESSION: CLEANING

8522 iP Study of droplet spray impact on a photomask surface [8522-42]

S. Singh, U. Dietze, SÜSS MicroTec Inc. (United States); P. Dress, SÜSS MicroTec Photomask Equipment GmbH \& Co. KG (Germany)

$85221 Q \quad$ Study of the durability of the Ru-capped MoSi multilayer surface under megasonic cleaning [8522-60]

H. Kurtuldu, A. Rastegar, M. House, SEMATECH (United States)

POSTER SESSION: MASK INSPECTION AND REPAIR

8522 IR Linear time EUV blank defect mitigation algorithm considering tolerance to inspection inaccuracy [8522-62]

Y. Du, Univ. of Illinois at Urbana-Champaign (United States); H. Zhang, Synopsys Inc. (United States); M. D. F. Wong, Univ. of Illinois at Urbana-Champaign (United States)

8522 is Efficient simulation of EUV multilayer defects with rigorous data base approach [8522-63]

P. Evanschitzky, F. Shao, A. Erdmann, Fraunhofer-Institut für Integrierte System und

Bavelementetechnologie (Germany)

$85221 \mathrm{U}$ Fiducial mark requirements from the viewpoints of actinic blank inspection tool for phase defect mitigation on EUVL mask [8522-65]

T. Murachi, T. Amano, S. H. Oh, EUVL Infrastructure Development Ctr., Inc. (Japan)

8522 IV EUVL mask inspection at Hydrogen Lyman Alpha [8522-66]

T. S. Jota, T. D. Milster, College of Optical Sciences, The Univ. of Arizona (United States)

8522 IW EUV mask blank defect avoidance solutions assessment [8522-67]

A. Elayat, P. Thwaite, S. Schulze, Mentor Graphics Corp. (United States) 
8522 IX Backside defect printability for contact layer with different reticle blank material [8522-68] G. Ning, GLOBALFOUNDRIES Dresden (Germany) and GLOBALFOUNDRIES Malta (United States); C. Holfeld, D. Fischer, GLOBALFOUNDRIES Dresden (Germany); P. Ackmann, GLOBALFOUNDRIES Malta (United States); A. Holfeld, K. Kurth, GLOBALFOUNDRIES Dresden (Germany); M. Sczyrba, Advanced Mask Technology Ctr. GmbH Co. KG (Germany); T. Hertzsch, R. Seltmann, GLOBALFOUNDRIES Dresden (Germany); A. Ho, F. H. GN, GLOBALFOUNDRIES Singapore Pte. Ltd. (Singapore)

$85221 \mathrm{Y}$ Key issues in automatic classification of defects in post-inspection review process of photomasks [8522-70]

M. Pereira, M. Maji, R. R. Pai, S. B. V. R., S. R., P. Patil, SoftJin Technologies Pvt. Ltd. (India)

852212 RDMS: a Windows and tablet PC based reticle defect search database with AHDC for interconnected mask and wafer fabs [8522-71]

S. Munir, G. Qidwai, Reticle Labs. LLC (United States)

852220 Status of the AIMS EUV Project [8522-72]

A. Garetto, J. H. Peters, S. Perlitz, U. Matejka, Carl Zeiss SMS (Germany); D. Hellweg, M. Weiss, Carl Zeiss SMT (Germany)

\section{POSTER SESSION: MASK DATA PREPARATION}

852221 Double patterning for 20nm and beyond: design rules aware splitting [8522-73] T. Desouky, Mentor Graphics Egypt (Egypt); D. Abercrombie, Mentor Graphics Corp. (United States); H. Kim, S. Choi, SAMSUNG Electronics Co., Ltd. (Korea, Republic of)

852222 Placement-aware decomposition of a digital standard cells library for double patterning lithography [8522-74]

A. G. Wassal, Cairo Univ. (Egypt); H. Sharaf, S. Hammouda, Mentor Graphics Egypt (Egypt)

852223 Novel customized manufacturable DFM solutions [8522-76]

M. Lu, C.-S. Zhou, Y. Tian, S.-M. Goh, S.-F. Quek, H.-M. Lam, J. Zhang, GLOBALFOUNDRIES Singapore (Singapore)

852224 Efficient Boolean and multi-input flow techniques for advanced mask data processing [8522-78]

D. Salazar, B. Moore, J. Valadez, Synopsys, Inc. (United States)

852225 Split-it!: from litho etch litho etch to self-aligned double patterning decomposition [8522-79]

Y. A. Badr, A. G. Wassal, Cairo Univ. (Egypt); S. Hammouda, Mentor Graphics (Egypt)

\section{POSTER SESSION: METROLOGY}

852226 Photomask quality evaluation using lithography simulation and precision SEM image contour data [8522-81]

T. Murakawa, N. Fukuda, S. Shida, T. Iwai, J. Matsumoto, T. Nakamura, Advantest Corp. (Japan); K. Hagiwara, S. Matsushita, D. Hara, D2S K.K. (Japan); A. Adamov, D2S, Inc. (United States) 
852228 Study for compensation of unexpected image placement error caused by VSB mask writer deflector [8522-86]

H.-J. Lee, M.-K. Choi, S.-Y. Moon, H.-K. Cho, SAMSUNG Electronics Co., Ltd. (Korea, Republic of); J. Doh, SAMSUNG Electronics Co., Ltd. (Korea, Republic of) and Hanyang Univ. (Korea, Republic of); J. Ahn, Hanyang Univ. (Korea, Republic of)

852229 Proximity effect correction optimizing image quality and writing time for an electron multibeam mask writer [8522-87]

T. Klimpel, Synopsys GmbH (Germany); J. Klikovits, IMS Nanofabrication AG (Austria);

R. Zimmermann, M. Schulz, Synopsys GmbH (Germany); A. Zepka, Synopsys, Inc. (United States); H.-J. Stock, Synopsys GmbH (Germany)

8522 2A Evaluation of CP shape correction for e-beam writing [8522-88]

M. Takizawa, K. Bunya, H. Isobe, H. Komami, K. Abe, M. Kurokawa, A. Yamada, K. Sakamoto, T. Nakamura, Advantest Corp. (Japan); K. Kuwano, M. Tateishi, D2S K.K. (Japan); L. Chau, D2S, Inc. (United States)

\section{POSTER SESSION: PATTERNING}

$85222 \mathrm{H}$ Impact of EUV photomask line-edge roughness on wafer prints (Second Place, Best Poster Award) [8522-96]

Z. J. Qi, E. Gallagher, IBM Microelectronics (United States); Y. Negishi, Toppan Photomasks, Inc. (United States); G. McIntyre, A. Zweber, IBM Microelectronics (United States); T. Senna, S. Akutagawa, T. Konishi, Toppan Photomasks, Inc. (United States)

8522 21 Dry etching technologies for reflective multilayer [8522-105]

Y. lino, M. Karyu, H. Ita, Y. Kase, T. Yoshimori, M. Muto, M. Nonaka, M. Iwami, Shibaura Mechatronics Corp. (Japan)

\section{POSTER SESSION: PROCESS}

8522 2J Reticle and wafer CD variation for different dummy pattern [8522-97]

G. Ning, GLOBALFOUNDRIES Dresden (Germany) and GLOBALFOUNDRIES Malta (United States); C. Buergel, Advanced Mask Technology Ctr. GmbH Co. KG (Germany); P. Ackmann, GLOBALFOUNDRIES Malta (United States); M. Staples, GLOBALFOUNDRIES Dresden (Germany); T. Thamm, C. T. Lim, A. Leschok, S. Roling, GLOBALFOUNDRIES Dresden (Germany); A. Zhou, F. H. GN, GLOBALFOUNDRIES Singapore Pte. Ltd. (Singapore); F. Richter, GLOBALFOUNDRIES Dresden (Germany)

8522 2K Bimetallic grayscale photomasks for micro-optics fabrication using dual wavelength laser writing techniques [8522-98]

R. Qarehbaghi, G. H. Chapman, Simon Fraser Univ. (Canada) 
8522 2L CD uniformity improvement through elimination of hardware influences on post-exposure bake [8522-99]

J. H. Lim, S. H. Woo, E. S. Park, S. P. Kim, D. G. Yim, Hynix Semiconductor Inc. (Korea, Republic of); O. Katada, SUSS MicroTec K.K. (Japan); T. Wähler, P. Dress, SÜSS MicroTec Photomask Equipment (Germany); U. Dietze, SüsS MicroTec Inc. (United States)

8522 2M Photomask etch: addressing the resist challenges for advanced phase-shift and binary photomasks [8522-100]

M. Grimbergen, M. Chandrachood, K. YU, T. Y. B. Leung, A. Sabharwal, A. Kumar, Applied Materials, Inc. (United States)

\section{POSTER SESSION: SIMULATION}

852220 Hotspot classification based on higher-order local autocorrelation [8522-102]

B. Lin, Z. Shi, Zhejiang Univ. (China); Y. Chen, Anchor Semiconductor, Inc. (United States)

8522 2P Proximity effect correction parameters for patterning of EUV reticles with Gaussian electron beam lithography [8522-103]

A. Lyons, J. Hartley, SUNY Univ. at Albany (United States)

$85222 Q \quad$ Nanoparticle detection limits of TNO's Rapid Nano: modeling and experimental results [8522-106]

P. van der Walle, P. Kumar, D. Ityaksov, R. Versulis, D. J. Maas, O. Kievit, J. Janssen,

J. C. J. van der Donck, TNO (Netherlands)

Author Index 


\title{
Conference Committee
}

\author{
Conference Chairs
}

Frank E. Abboud, Intel Corporation (United States)

Thomas B. Faure, IBM Corporation (United States)

\section{Conference Program Committee}

Ronald R. Bozak, RAVE LLC (United States)

William H. Broadbent, KLA-Tencor Corporation (United States)

Peter D. Buck, Toppan Photomasks, Inc. (United States)

Russell B. Cinque, JEOL USA Inc. (United States)

Glenn R. Dickey, Shin-Etsu MicroSi, Inc. (United States)

Uwe Dietze, Suss MicroTec AG (Germany)

Aki Fujimura, D2S, Inc. (United States)

Emily E. Gallagher, IBM Corporation (United States)

Brian J. Grenon, Grenon Consulting, Inc. (United States)

Naoya Hayashi, Dai Nippon Printing Company, Ltd. (Japan)

Mark T. Jee, HOYA Corporation USA (United States)

Rik Jonckheere, IMEC (Belgium)

Bryan S. Kasprowicz, Photronics, Inc. (United States)

Byung-Gook Kim, SAMSUNG Electronics Company, Ltd. (Korea,

Republic of)

Shy-Jay Lin, Taiwan Semiconductor Manufacturing Company Ltd.

(Taiwan)

Wilhelm Maurer, Infineon Technologies AG (Germany)

M. Warren Montgomery, College of Nanoscale Science \&

Engineering (United States)

Thomas H. Newman, Micronic Laser Systems Inc. (United States)

Hiroshi Nozue, NuFlare Technology, Inc. (Japan)

Linyong Pang, Luminescent Technologies, Inc. (United States)

Emmanuel Rausa, Plasma-Therm LLC (United States)

Douglas J. Resnick, Molecular Imprints, Inc. (United States)

Thomas Scherübl, Carl Zeiss SMS GmbH (Germany)

Steffen Schulze, Mentor Graphics Corporation (United States)

Robert J. Socha, ASML US, Inc. (United States)

Anna Tchikoulaeva, Lasertec U.S.A., Inc. Zweigniederlassung

Deutschland (Germany)

Jacek K. Tyminski, Nikon Research Corporation of America (United States)

Banqiu Wu, Applied Materials, Inc. (United States)

Stefan Wurm, SEMATECH North (United States) 
Invited Session

Frank E. Abboud, Intel Corporation (United States)

Thomas B. Faure, IBM Corporation (United States)

Patterning

Jacek K. Tyminski, Nikon Precision Inc. (United States)

Robert J. Socha, ASML US, Inc. (United States)

Metrology

Thomas Scherübl, Carl Zeiss SMS GmbH (Germany)

Peter D. Buck, Toppan Photomasks, Inc. (United States)

Mask Inspection and Repair I

Emily E. Gallagher, IBM Corporation (United States)

William H. Broadbent, KLA-Tencor Corporation (United States)

Material and Process

Banqiu Wu, Applied Materials, Inc. (United States)

Byung-Gook Kim, SAMSUNG Electronics Company, Ltd. (Korea, Republic of)

Mask Data Preparation I

Aki Fujimura, D2S, Inc. (United States)

Steffen F. Schulze, Mentor Graphics Corporation (United States)

Simulation and Modeling

Linyong Pang, Luminescent Technologies (United States)

M. Warren Montgomery, College of Nanoscale Science \& Engineering (United States)

Cleaning/Contamination/Haze

Brian J. Grenon, Grenon Consulting, Inc. (United States)

Anna V. Tchikoulaeva, Lasertec U.S.A., Inc. Zweigniederlassung Deutschland (Germany)

Source/Mask Optimization

Wilhelm Maurer, Infineon Technologies AG (Germany)

Thomas H. Newman, Micronic Laser Systems Inc. (United States)

Mask Long-Term Durability

Naoya Hayashi, Dai Nippon Printing Company, Ltd. (Japan)

Stefan Wurm, SEMATECH North (United States) 
Mask Pattern Generators

Hiroshi Nozue, NuFlare Technology, Inc. (Japan)

Russell B. Cinque, JEOL USA Inc. (United States)

Mask Inspection and Repair II

Ron R. Bozak, RAVE LLC (United States)

Uwe Dietze, SUSS MicroTec Inc. (United States)

\section{Session Panel Moderators}

Special Session Panel Discussion

Thomas B. Faure, IBM Corporation (United States)

Robert J. Socha, ASML US, Inc. (United States) 
Proc. of SPIE Vol. $8552855201-16$

Downloaded From: https://www.spiedigitallibrary.org/conference-proceedings-of-spie on 26 Apr 2023 Terms of Use: https://www.spiedigitallibrary.org/terms-of-use 


\section{Introduction}

This proceedings volume contains accepted papers from the SPIE conference on Photomask Technology 2012. The conference was arranged through the Bay Area Chrome Users Society (BACUS) and held as part of the $32^{\text {nd }}$ International Symposium on Photomask Technology 10-13 September 2012 in Monterey, California, United States of America.

This year's conference included three invited papers in addition to the annual invited Mask Industry Assessment presentation by SEMATECH, and the best paper by Photo Mask Japan (PMJ) 2012 and a summary of the PMJ 2012 Panel Discussion.

The conference presentations this year were organized in a single track format, allowing every participant the time to get a comprehensive overview of all of the subjects in the field of Photomask. We added electronic voting for best oral paper and a subset of the program committee members selected the best posters. In addition, a Mask Long-Term Durability session was added to encourage focus on this emerging topic. Patterning and Mask Pattern Generators where split into two distinct sessions to allow a more focused discussion on both topics.

One additional highlight of the conference was the keynote presentation by John Chen from NVIDIA. Dr. Chen presented on the importance of the mask and the key role it plays in the lithography process, "The mask has a huge impact if it is not done right". Further he quantified "right" by introducing the three P's: Performance, Precision and Perfection. Showing such linkage between the mask quality and the wafer/chip performance from the view point of the end user was refreshing as it allowed the audience to see how their work is important and valued. In his talk he showed, as an example, the impact of a $1 \mathrm{~nm}$ CD variation on the end products. Dr. Chen concluded by reaffirming that the mask and the mask industry are key to the continuation of Moore's Law.

The technical focus of this year's conference has been how to get ready with two technologies in parallel, the arrival of EUV in the mainstream of Mask Technology and the complex extension of Optical Lithography. This is a challenge for the Mask industry as both Litho solutions depend heavily on the Mask readiness. In the 2011 panel discussion titled: "Is it too late for panic?...EUV is real!", the industry consensus was that the mask will not be an obstacle. This year's special session was Optical Extensions. Optical Lithography continues to extend and has surpassed everyone's expectations. 
The ability to pattern geometries 10x smaller than the wavelength may need to go a step further! The special session was titled: "Will optical patterning solutions be ready if EUV lithography is delayed?" The session was chaired by Bob Socha and Tom Faure and revealed key mask capabilities and technology plans that can meet that challenge. A group of our industry's top experts were assembled for the discussion:

- Allen Gabor, Senior Patterning Program Manager in Advanced Lithography, IBM Corp.

- Aki Fujimura, Chairman and CEO D2S Inc.

- Yuri Granik, Chief Scientist, Design to Silicon Division, Mentor Graphics.

- Yoshio Kawai, Deputy General Manager, ShinEtsu New Functional Materials Research Center

- Albert Wang, Senior Staff Process Engineer, SanDisk Corp.

- Franklin Kalk, Executive Vice President and Chief Technology Officer, Toppan Photomasks Inc.

We thank all of the participants and especially the authors for providing and discussing their insights. We also thank all of the members of the program committee for their hard work in helping to make this year's conference a success through their efforts which ranged from reviewing abstracts to chairing sessions. Our sponsors also deserve special thanks for their continued support of Photomask Technology. The SPIE organization has our gratitude for their tireless efforts in organizing the conference and ensuring that things ran smoothly as well as their efforts to provide a timely publication of these proceedings. And a special thanks to the BACUS entertainment team who did a fantastic job in bringing back the traditional BACUS entertainment themes!

We hope you find the papers contained in this volume of proceedings to be informative and helpful in your professional endeavors.

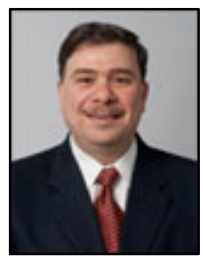

Frank E. Abboud Intel Corporation Symposium Chair

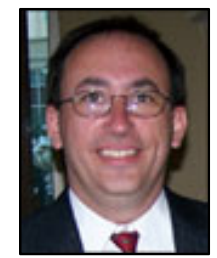

Thomas B. Faure

IBM Corporation Symposium Cochair 
Thursday Special Session Panel Discussion

\section{Will optical patterning solutions be ready if EUV lithography continues to be delayed?}

Panel Moderators: Thomas B. Faure, IBM Corporation, Robert J. Socha, ASML US, Inc.

If EUV lithography is delayed, lessons learned of extending ArF lithography at the $20 \mathrm{~nm}$ and $14 \mathrm{~nm}$ nodes can be applied to the $10 \mathrm{~nm}$ and $7 \mathrm{~nm}$ nodes. Most likely the shrink will be enabled by integration of all of the aspects needed to decrease the cost per transistor. These aspects include the mask, the lithography, the EDA, and the design. This panel will focus on the interaction of the mask with lithography, EDA, and design.

In order to focus on the interaction of the mask with integration, the panel will discuss an overview of optical patterning solutions for the $10 \mathrm{~nm}$ and $7 \mathrm{~nm}$ nodes for both logic and memory. For these nodes, the mask making challenges will impact the optical lithography extensions which will test and impose limitations on the design and computational lithography ground rules. Furthermore, these issues will require changes in the mask making infrastructure and may require more aggressive mask strategies (PSM rather than binary) and may require a multiple beam mask writer. In order to answer these questions, the panel will discuss these challenges through a diverse group of experts from the mask industry, from the lithography community, from the EDA industry, and from the fabless companies which are end users of the mask.

Panelists:

Allen Gabor, Senior Patterning Program Manager in Advanced Lithography, IBM Corporation

Aki Fujimura, Chairman and CEO, D2S Inc.

Yuri Granik, Chief Scientist, Design to Silicon Division, Mentor Graphics Corporation

Yoshio Kawai, Deputy General Manager, New Functional Materials Research Center, ShinEtsu Chemical Company Ltd.

Tuan Pham, Director of Flash Process \& Device Technology, SanDisk Corporation Geoffery Yeap, Vice President of Technology, Qualcomm Inc.

Franklin Kalk, Executive Vice President and Chief Technology Officer, Toppan Photomasks Inc.

* For information about the BACUS technical group go to: www.SPIE.org/BACUSHome 
Proc. of SPIE Vol. $8552855201-20$

Downloaded From: https://www.spiedigitallibrary.org/conference-proceedings-of-spie on 26 Apr 2023 Terms of Use: https://www.spiedigitallibrary.org/terms-of-use 